\title{
Improvement of a CHO Fed-Batch Process by Fortifying with Plant Peptones
}

\author{
Yun Jiang, Erik Svensson, and Véronique Chotteau
}

\begin{abstract}
A serum-free fed-batch process was developed for production of a human monoclonal antibody in Chinese hamster ovary (CHO) cells based on Biovitrum's proprietary low protein serum-free medium without animal derived components (BVT4). The cells were fed with glucose, glutamine and Biovitrum's proprietary low protein serum-free feed medium without animal derived components enriched with amino acids, vitamins, metal traces, peptones, and biosynthesis precursors. To improve the performance of the fed-batch process, we developed the use of plant peptones by studying the dose and timing of the peptone feeding. Different doses of peptone cocktail and amino acid cocktail, as well as different combinations of peptone and amino acid cocktails were first screened in $50 \mathrm{ml}$ filter tubes on an AgCell shaker table. The best combinations were then assessed in spinner and $3 \mathrm{~L}$ bioreactor cultures. To reinforce our findings, the antibody-producing CHO cells were adapted to a disclosed serum-free medium DMEM/F12 and the beneficial effects of peptones were confirmed in a fed-batch process based on the DMEM/F12 serum-free medium.

Only parts of the poster results are presented and discussed here.
\end{abstract}

Keywords Peptone $\cdot \mathrm{CHO} \cdot$ Fed-batch $\cdot$ Medium $\cdot$ Chinese Hamster Ovary

\section{Materials and Methods}

Antibody producing CHOK1 cell line; BVT4 basal medium supplemented with $5 \mathrm{~g} / \mathrm{L}$ peptone cocktail, peptone cocktail: $50 \%$ of Hypep7504 and 50\% of Hypep 7401 from Kerry; amino acid cocktail: RPMI 1640 amino acid solution enriched in asparagine, serine, and tryptophan; Cell counting: Cedex INNOVATIS; antibody titer: Sandwich ELISA; Glucose/Lactate/Glutamine/Glutamate concentrations:

Y. Jiang (凶)

Biovitrum, Biopharmaceutical Process Development, SE-112 76, Stockholm, Sweden

e-mail: veronique.chotteau@biovitrum.com 
YSI 2700; pH: Blood gas analyzer ABL; amino acids analysis: Cation exchange chromatography HPLC; osmolarity: Roebling Automatic osmometer.

\section{Results}

\subsection{Experiment 1: Neutralization of the Toxic Effect from Amino Acid Over-Feeding by Peptones Addition}

Experimental design: cultivation in four fed spinners in BVT4 medium, \#1, \#2, \#3, and \#4. Spinner \#1 was fed every other day with several components: glucose, glutamine and concentrated feed medium consisting of the basal medium enriched in vitamins, metals and biosynthesis precursors (feed medium only). Spinner \#2 was fed as \#1, as well as with the peptone cocktail at a total dose of $1.2 \mathrm{~g} / \mathrm{L}$ every other day (feed medium + peptones). Spinner \#3 was fed as \#1, as well as a cocktail of amino acid (feed medium + amino acid cocktail). Spinner \#4 was fed as \#1, as well as the peptone and amino acid cocktails with the same doses as spinner \#2 and \#3, respectively (feed medium + peptones + amino acid cocktail). A batch cultivation in the same basal medium was performed as a reference.

Results: The viable cell number and viability increased when peptones were fed to the culture (\#2 vs. \#1). The viable cell number and viability decreased when the amino acid cocktail was fed to the culture (\#3 vs. \#1), indicating a toxic effect from the amino acid feeding. The viable cell number and viability were improved when both the amino acid cocktail and the peptones were fed to the culture (\#4 vs. \#3), indicating that addition of the peptones partially neutralized the toxic effect from amino acid over-feeding. Spinner \#2 gave the highest antibody production, followed by \#1, \#4, \#3 and finally the batch spinner.

\subsection{Experiment 2: Dose Study in $50 \mathrm{ml}$ Filtered Tubes}

Experimental design: Duplicate cultures (4 mL) were set up in BVT4 medium in $50 \mathrm{~mL}$ agitated filtered tubes to study the dose of peptone feeding. Extra amino acid feeding was also tested by adding amino acid cocktail at two doses. Glucose, glutamine, peptone cocktail (peptone) and/or amino acid cocktail ( $a a$ ) were fed every other day from day 2, see Table 1. 2.9 mL BVT feed medium were fed every third day from day 3.

Table 1 Experiment 2 tube scheme

\begin{tabular}{llllllll}
\hline & $\# 1$ & $\# 2$ & $\# 3$ & $\# 4$ & $\# 5$ & $\# 6$ & batch \\
\hline $\begin{array}{l}\text { peptone } \\
\text { aa }\end{array}$ & $\mathrm{P} 1$ & $\mathrm{P} 2$ & $/$ & $/$ & $\mathrm{P} 1$ & $\mathrm{P} 2$ & $/$ \\
\hline
\end{tabular}

peptone cocktail: $\mathrm{P} 1=0.8 \mathrm{~g} / \mathrm{L}$ days 2,4 and $0.4 \mathrm{~g} / \mathrm{L}$ days 6,$8 ; \mathrm{P} 2=1.6 \mathrm{~g} / \mathrm{L}$ days 2,4 and $0.8 \mathrm{~g} / \mathrm{L}$ days 6,8 ; $a$ a cocktail: $\mathrm{A} 1=0.4 \mathrm{~mL}$ days $2,4,6$ and $0.2 \mathrm{~mL}$ day $8 ; \mathrm{A} 2=1.6 \mathrm{~mL}$ days $2,4,6$ and $0.8 \mathrm{~mL}$ day 8 


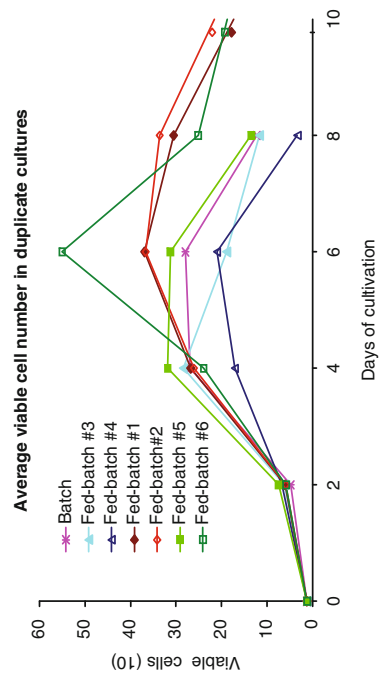

(\%) К‼!qu!

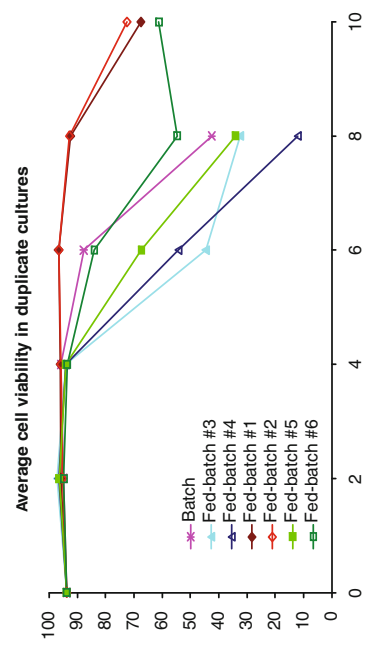

(\%) Кํ!!!qe!^

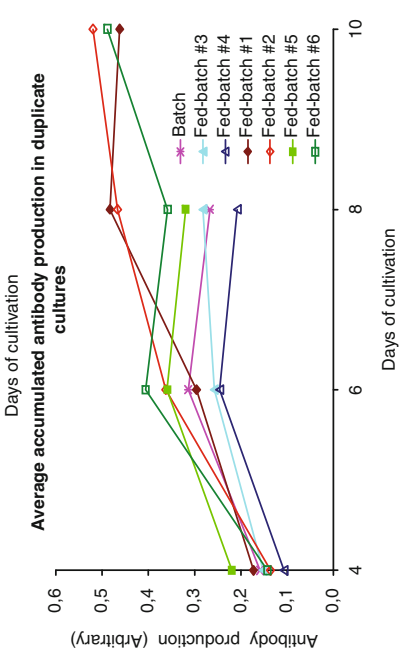

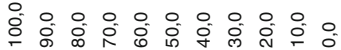
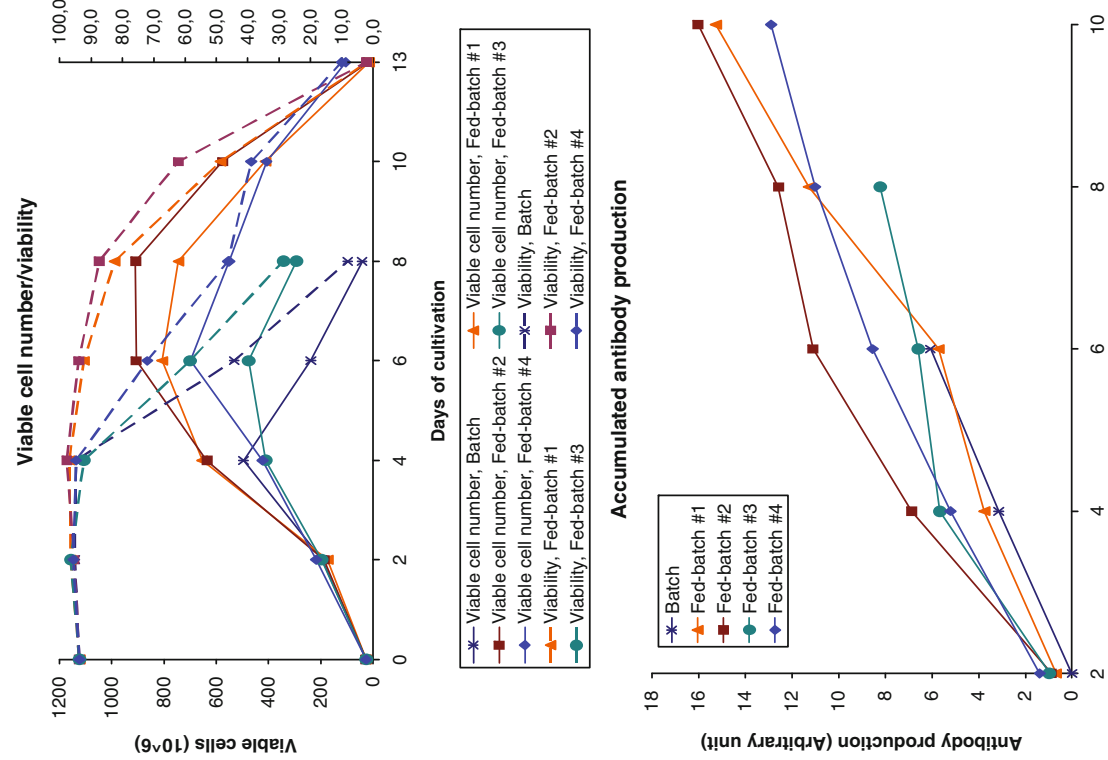

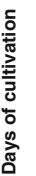

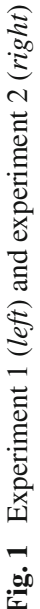


Results: The viable cell number, viability, and antibody production increased significantly in the cultures fed with peptones. Beneficial effects of the peptones could not be reproduced by the amino acid supplementation. Over-feeding amino acid was detrimental to cell growth and antibody production. Addition of peptones partially neutralized the toxic effect from amino acid over-feeding (Fig.1).

\section{Conclusion}

Feeding plant peptones significantly improved the cell growth, process longevity and productivity in a $\mathrm{CHO}$ fed-batch process based on Biovitrum's proprietary low protein serum-free medium without animal derived components. The beneficial effects of the peptones could not be reproduced by the amino acid supplementation. Feeding plant peptones partially neutralized the toxic effect from amino acids overfeeding, improving cell growth and productivity in an under-optimized fed-batch process. The beneficial effects of peptones were reinforced in a fed-batch process based on the disclosed DMEM/F12 serum-free medium.

Acknowledgments We thank the company Symphogen, Denmark, for providing us the antibodyproducing $\mathrm{CHO}$ cell line. A special thank to Dr. Anne Bondgaard Tolstrup and Dr. Sören Bregenholt for their kind support to get this permission and helping us in the publication release of this work. We also thank the company Kerry for providing us Kerry peptones. 